\title{
Tomographic radio occultation methods applied to a dense cubesat formation in low Mars orbit
}

\section{Journal Article}

Author(s):

Möller, Gregor (D); Ao, Chi O.; Mannucci, Anthony J.

Publication date:

2021-07

Permanent link:

https://doi.org/10.3929/ethz-b-000493222

Rights / license:

Creative Commons Attribution-NonCommercial-NoDerivatives 4.0 International

Originally published in:

Radio Science 56(7), https://doi.org/10.1029/2020rs007199 


\section{Radio Science}

\section{RESEARCH ARTICLE \\ 10.1029/2020RS007199 \\ Tomographic Radio Occultation Methods Applied to a Dense Cubesat Formation in Low Mars Orbit}

Key Points:

- Dense cubesat formations are a promising means to fill in gaps in the existing observation network

- Atmospheric tomography is an innovative method for the processing of overlapping cross-link radio occultation measurements

- Tomography derived products can provide valuable insight into the temperature and pressure structure in the lower Martian atmosphere

\section{Correspondence to: \\ G. Moeller, \\ gmoeller@ethz.ch}

\section{Citation:}

Moeller, G., Ao, C. O., \& Mannucci, A. J. (2021). Tomographic radio occultation methods applied to a dense cubesat formation in low Mars orbit. Radio Science, 56, e2020RS007199. https://doi. org/10.1029/2020RS007199

Received 20 SEP 2020

Accepted 7 JUN 2021

Plain Language Summary Satellite missions to Mars are crucial for monitoring the

\author{
Gregor Moeller $^{1,2}$ (D), Chi O. Ao ${ }^{1}$ (D), and Anthony J. Mannucci ${ }^{1}$ (D) \\ ${ }^{1}$ NASA Jet Propulsion Laboratory, California Institute of Technology, Pasadena, CA, USA, ${ }^{2}$ Now at: Institute of Geodesy \\ and Photogrammetry, ETH Zürich, Zurich, Switzerland
}

\begin{abstract}
The atmospheric measurements made by the eight Mars orbiters in operation (as of April 2021) significantly improved our understanding of the Martian weather and climate. However, while some of the existing Mars orbiters will reach their lifetime, innovative and cost-effective observation concepts are requested - not only to guarantee continued observation but also to address potential gaps in the existing observing network. Inspired by the success of the two MarsCube One satellites we have established an observation concept, which is based on a series of cubesats, carried to Mars and injected into a low Mars orbit in a so-called string-of-pearls formation. Over the lifetime of the cubesats of about one Martian year, a series of radio occultation (RO) experiments will be carried out with up to 180 partially overlapping RO measurements per day. For processing of the unique set of observations, tomographic principles are applied to the RO measurements for the reconstruction of high-resolution twodimensional temperature and pressure fields of the lower Martian atmosphere. Unlike the standard Abel transform approach, the derived products are not dependent on spherical symmetry assumptions. Thus, insights into various unresolved atmospheric phenomena are obtained - especially of those which are characterized by distinct horizontal gradients in pressure and temperature, for example, as observed at the day-night terminator, during dust storms, or over complex terrain.
\end{abstract} atmospheric state and to derive valuable information about the weather and climate on our red fellow planet. When traveling through the atmosphere, the radio links between orbiting satellites are delayed and the frequency shifts can be used to carefully study the atmospheric processes in detail. However, the existing Mars orbiters are not designed for cross-link measurements between the orbiters and thus, the number of radio observations is limited. In order to overcome current limitations, we present a new observation concept, which is based on four cubesats, deployed into in a so-called "string-of-pearls" formation around Mars. The established constellation will allow for 180 globally distributed measurement series per day and each series opens the ability to study horizontal and vertical structures in the Martian atmosphere with fine resolution. A new processing strategy based on tomographic principles applied to the radio observations will allow to further increase the horizontal resolution. The obtained products will give an insight into various unresolved atmospheric phenomena, for example, at the day-night terminator, during dust storms or at the edge of the polar ice caps.

\section{Introduction}

Since the very beginning of planetary exploration, communication links between the Earth and spacecraft are used not only for data transfer but also to "examine important properties of planetary atmospheres [...] by carefully studying small changes in the radio signal's frequency" (Asmar et al., 2019) when the spacecraft occults behind the planet. The first radio science experiment through the Martian atmosphere was carried out during the flyby of the Mariner 4 spacecraft in 1965 (Kliore et al., 1965). Since then, almost every mission to Mars, either flyby or orbiter mission, has been used for planetary radio occultation (RO) experiments to obtain a better insight into the atmospheric processes on Mars.

The eight Mars orbiters in operation (as of April 2021) are equipped with S-band (2.3 GHz), X-band $(8.4 \mathrm{GHz})$ or Ka-band $(31.8 \mathrm{GHz})$ communication links suitable for radio science experiments. Nevertheless, the typical Earth-to-spacecraft geometry for planetary RO results in limited latitude and local time distribution for the profiles, see Withers et al. (2020). In addition, longer observation gaps appear, for example, 
when the orbital plane of the spacecraft is perpendicular to the Earth-Mars line or if the Earth-Mars line is too close to the Sun.

In order to further increase the number of observations, Ao et al. (2015) have carried out a series of crosslink occultation experiments using the ultra high frequency (UHF)-band $(0.4 \mathrm{GHz})$ Electra transceivers of Mars Odyssey (ODY) and Mars Reconnaissance Orbiter (MRO). The UHF communication link is designed for proximity communications with the Mars landers and rovers. During regular relay service between ODY and a ground asset, MRO was eavesdropping to the UHF signal of ODY and recorded the in-phase and quadrature components in open-loop tracking mode. The analysis of the phase measurements revealed that the signal-to-noise ratio of the received UHF signal and the clock stability is sufficient for RO studies of the electron density in the ionosphere between 50 and $200 \mathrm{~km}$ altitude.

Motivated by the success of the first cross-link experiments, further cross-link measurements are being planned, for example, between Mars Express and the ExoMars Trace Gas Orbiter (Hakan Svedhem, personal communication, Sep 2019). Various studies (Asmar et al., 2016; Tellmann et al., 2019; Mannucci et al., 2015) confirm that cross-link RO can produce high signal-to-noise ratio of the received signal, and further increase the planetary-scale distribution of the RO measurements. Though, if cross-link RO purely relies on existing Mars orbiters, its full potential cannot be exploited due to the limitations in radio frequency and orbital geometry. For example, the single-frequency UHF link does not allow for separation of ionospheric and neutral atmospheric effects. Furthermore, standard processing strategies based on the Abel transform do not allow for resolving horizontal features in the atmosphere (Fjeldbo et al., 1971; Hinson et al., 1999; Withers et al., 2014). To overcome these limitations, in the following we present a new observation concept based on tomographic RO methods - applied to a dense cubesat formation in low Mars orbit. Section 2 introduces the concept of dense cubesat formations. Section 3 focuses on the spatio-temporal distribution of the expected observations. Section 4 addresses the processing of the RO signals using tomographic principles. The tomography case study itself is described in Section 5. A conclusion and outlook will be provided in Section 6.

\section{Concept of Dense Cubesat Formations}

For our study, we assume a series of four cubesats, carried to Mars and injected into a low Mars orbit as secondary payload on a larger orbiter. A similar concept based on cubesats flying in close formation has recently been proposed for an Earth explorer mission (Turk et al., 2019). The advantage from such configuration is that we can get simultaneous RO observations that are closely located.

Figure 1 highlights the possible deployment plan for a cubesat formation in low Mars orbit. Although the observation concept is not particularly dependent on a specific main orbiter, we selected ESA's Mars Sample Return (MSR) orbiter (Joffre et al., 2018) as a potential candidate - primarily because detailed aerocapture studies for cubesats are not available yet and the MSR orbiter would be one of the next possibilities to deploy cubesats into a low Mars orbit.

For the four cubesats, we suggest a so-called "string-of-pearls" formation (Tan et al., 2002). It provides the necessary observation geometry for sensing meso- to small-scale structures in the Martian atmosphere (see Section 3 for details). After deployment of the cubesats, the main orbiter carries out a series of maneuvers to reach its final orbit. The radio link between main orbiter and each of the four cubesats can be used to provide RO measurements. In the following, the orbit geometry and the individual components of the observation concept are described in detail.

\subsection{Selection of Orbits}

The orbital elements of the main orbiter are based on the numbers provided by Joffre et al. (2018), except for the inclination. Instead of $40^{\circ}$ we propose an inclination of $60^{\circ}$, which enables nearly global coverage for limb sounding and a reasonable nodal precession rate $(\dot{\Omega})$ 


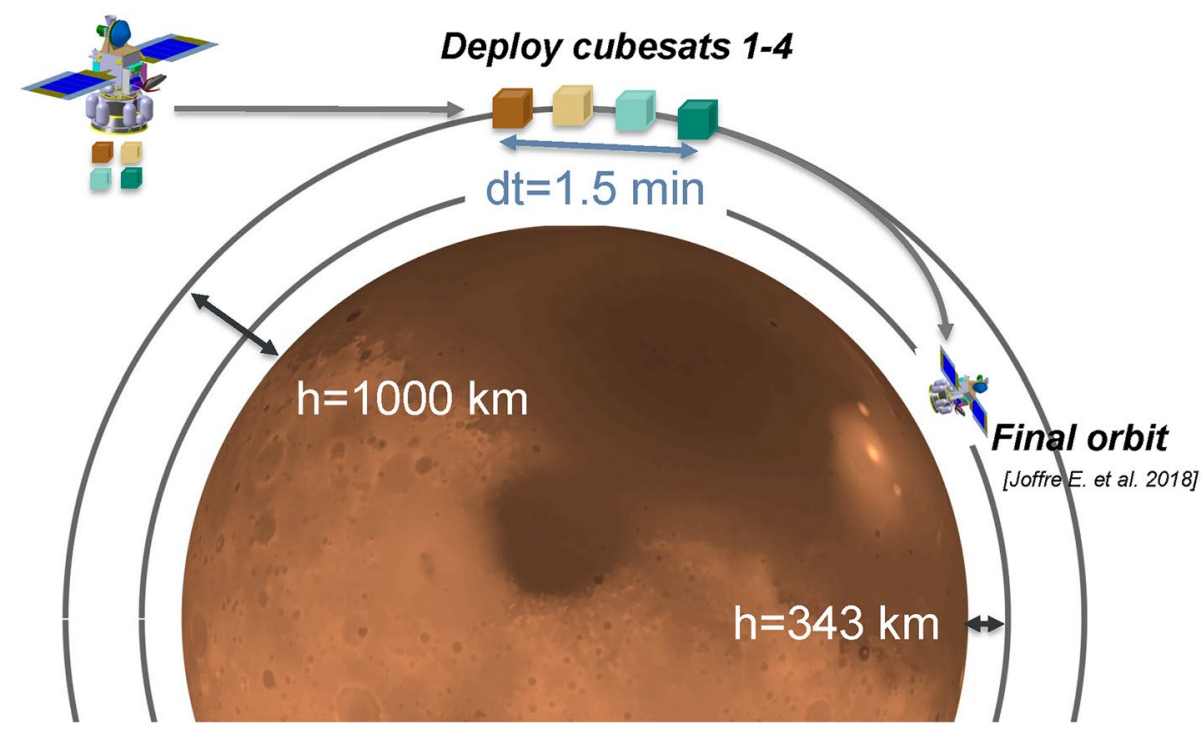

Figure 1. Cubesat deployment plan. The four cubesats are carried to Mars by a main orbiter and deployed into a socalled string-of-pearls formation.

$$
\dot{\Omega}(a, i)=\frac{-3}{2} \cdot n \cdot J_{2} \cdot \frac{R^{2}}{\left(a \cdot\left(1-e^{2}\right)\right)^{2}} \cdot \cos (i),
$$

where $n=\sqrt{G M / a^{3}}$ is the mean motion of the spacecraft, $a=R+h$ is the semi-major axis, $e$ is the eccentricity, and $i$ is the inclination of the orbital plane, with areocentric gravitational constant $G M=42,828.375214 \mathrm{~km}^{3} /$ $\mathrm{s}^{-2}$, mean Mars radius $R=3,389.5 \mathrm{~km}$ and spacecraft altitude $h$. The term $J_{2}=1.96045 \times 10^{-3}$ of the geopotential compensates for the non-sphericity of Mars (higher order terms are not considered). Assuming a spacecraft altitude of $343 \mathrm{~km}$ and an inclination of $60^{\circ}$, the nodal precession of the main orbiter works out to be $-5.2^{\circ}$ per solar day on Mars (sol).

For the four cubesats, we assume the same inclination as for the main orbiter. Besides, we propose an altitude of $1,000 \mathrm{~km}$ (orbital period of $2 \mathrm{~h} 27 \mathrm{~min}$ ), which seems to be beneficial due to several reasons. First, the selected orbit is not in resonance with the rotation rate of Mars $(24.659722 \mathrm{~h} / \mathrm{rev})$ and therefore, guarantees good longitudinal coverage. Second, the resulting nodal precession of the cubesats $\left(-3.2^{\circ} / \mathrm{sol}\right)$ allows for sensing of the entire diurnal cycle within one Martian season. In addition, the duration of the RO events seems to be most beneficial for the processing using tomographic RO methods (see Section 4).

The spacing between the cubesats is set to $d M=1.2^{\circ}$. At an altitude of $1,000 \mathrm{~km}$ this corresponds to a temporal spacing of about $30 \mathrm{~s}$. For the observation concept, the cubesat spacing is an important parameter. It defines not only the time between the RO measurements but also the observation geometry, that is, the horizontal resolution and area covered (see Section 3 for further details). Over the mission lifetime of 1 Martian year, we expect that the four cubesats can be kept in formation with minor orbital corrections. In

Table 1

Orbital Elements of the Main Orbiter and the Four Cubesats

\begin{tabular}{|c|c|c|c|c|c|c|}
\hline Spacecraft & $\begin{array}{c}h \\
(\mathrm{~km})\end{array}$ & $\begin{array}{c}i \\
(\operatorname{deg})\end{array}$ & $M_{0}(\mathrm{deg})$ & $e$ & $\begin{array}{c}\dot{\Omega}(\mathrm{deg} / \\
\text { sol) }\end{array}$ & $\begin{array}{c}\text { Period } \\
\text { (hh:mm) }\end{array}$ \\
\hline Main orbiter & 343 & 60 & 0 & 0.0 & -5.2 & 01:55 \\
\hline Cubesats 1-4 & 1,000 & 60 & $0,1.2,2.4,3.6$ & 0.0 & -3.2 & $02: 27$ \\
\hline
\end{tabular}

From left to right: Spacecraft altitude $h$, inclination $i$, mean anomaly $M_{0}$, eccentricity $e$, nodal precession rate $\dot{\Omega}$ according to Equation 1 and orbital period $P$. contrast, the orbital drift of the main spacecraft is less critical, that is, a change of the orbital elements of the main orbiter due to gravitational and non-gravitational forces has little impact on the number and distribution of the RO measurements.

\section{Spatio-Temporal Distribution of Observations}

Based on the orbital elements listed in Table 1, all possible cross-link RO events between main orbiter and the four cubesats have been identified. In the following, the results are shown for a period of 150 sols (about 5 months). This period reflects approximately the duration of the 2018 

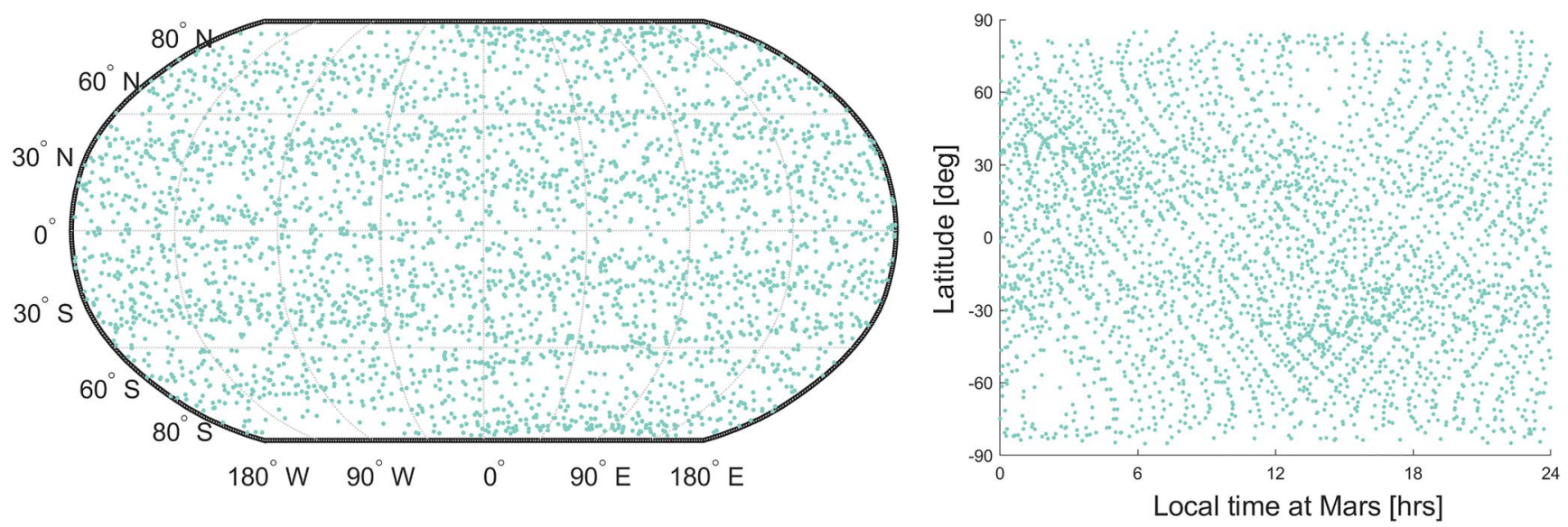

Figure 2. Left: Global distribution of radio occultation (RO) events as expected between main orbiter and each cubesat over a period of 150 sols. Each dot represents the tangent point of a RO event, that is, the point where the signal grazes the surface of Mars. Right: Latitude and local time coverage of the RO events for the same period of 150 sols.

global dust storm, which lasted from May-September 2018. Due to nodal precession, this is also the time needed to reach again the same orbit configuration ( $2.4 \%$ sol relative drift between orbital planes), that is, covers the possible observation geometries.

Figure 2 shows the global distribution of the 3,200 RO events as identified between main orbiter and cubesat 1 over the period of 150 sols. Due to the limb sounding geometry, a global distribution can be obtained in which not only the latitude bands between $60^{\circ} \mathrm{S}$ and $60^{\circ} \mathrm{N}$ are covered (as expected from the inclination of the orbits) but also the polar regions up to $85^{\circ}$ latitude. A similar distribution is expected between the main orbiter and the other three cubesats, which leads to a total of approximately 57,000 RO events within one Martian year. Due to the relatively close spacing, no additional occultation measurements are expected between the cubesats themselves. However, in the following it is shown why this configuration is beneficial.

The angle between the two orbital planes (main orbiter and cubesats 1-4) is constantly changing due to nodal precession. In consequence, the number of observations per sol, their global distribution but also the observation geometry of the RO events change too. In order to characterize the observation geometry and to better understand the temporal variations, in the following we will distinguish between two scenarios. In the first scenario, the orbital planes are perpendicular to each other and RO measurements are obtained in cross-track direction. In consequence, from the four cubesats in formation we obtain four ray paths which are widely parallel to each other, see Figure 3 left. In the second scenario (about 35 sols later) the orbital planes are aligned. As a consequence, RO measurements are obtained in flight direction. This provides a unique observation geometry, in which consecutive observations overlap, see Figure 3 right. With these
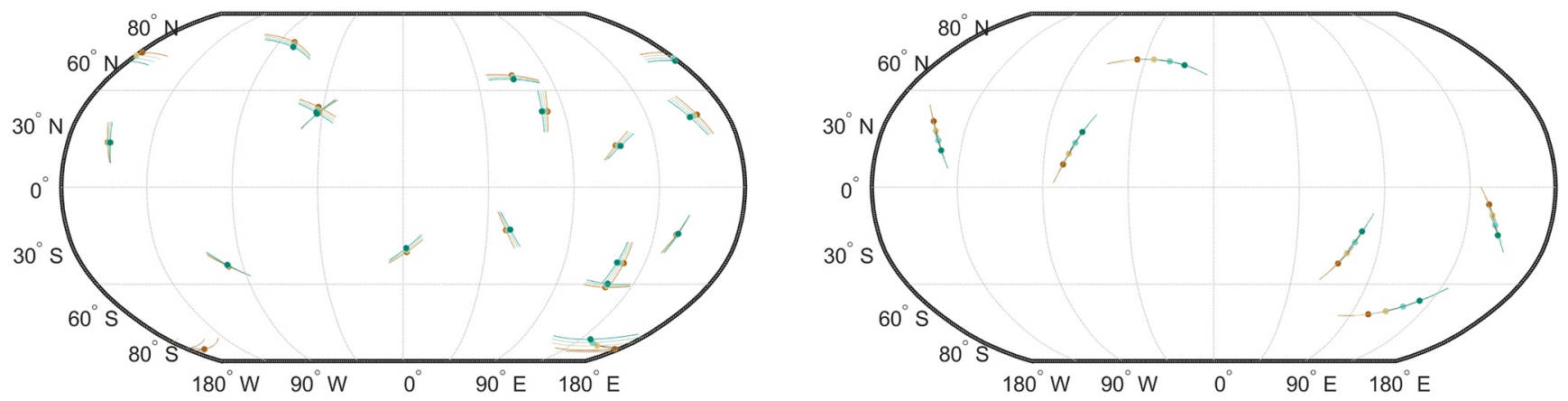

Figure 3. Geometry and distribution of radio occultation (RO) events - exemplary for two solar days on Mars. The left plot results from a $90^{\circ}$ angle between the orbital planes. The right plot is obtained, if the orbital planes are aligned. Each line represents a ray path between main orbiter and one cubesat, each dot the corresponding RO tangent point. 


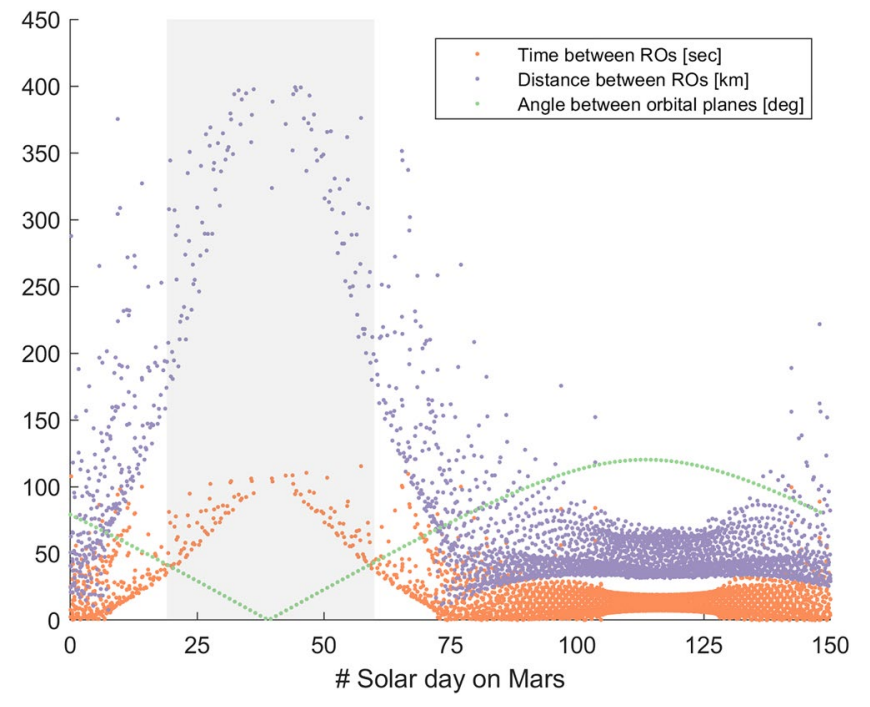

Figure 4. Selected parameters describing the clustering of radio occultation (RO) events. In case cubesat 1 becomes visible at the horizon, the "Time between ROs [sec]" indicates how long it takes until the second cubesat becomes visible for the main orbiter. The "Distance between ROs $[\mathrm{km}]$ " describes the horizontal distance between the RO events of cubesat 1 and 2. two configurations the distribution of observations is explained. All other cases can be described as a combination of the two scenarios.

In total, from the four cubesats we expect 20 to 180 cross-link ROs per day, which is more than currently recorded by eight operational orbiters in the typical Earth-spacecraft geometry. The varying number of events is explained by the changing observation geometry over the course of 150 sols. In case of aligned orbits (see Figure 3 right), all spacecraft fly in common directions with similar speed. This leads to a temporal clustering of RO events and longer observation gaps between the clusters.

According to Figure 3, the clustering of RO events seems to be beneficial for sensing of meso- to small-scale structures in the atmosphere. Especially due to the small temporal spacing of the four cubesats of about $30 \mathrm{~s}$, nearby regions in the atmosphere can be sensed almost simultaneously. In Figure 4, the spatio-temporal separation of consecutive ROs is highlighted - exemplary for the first two cubesats. Over the course of 150 sols, the time between two consecutive ROs varies between a few seconds, but can be up to $100 \mathrm{~s}$. In consequence, the horizontal distance between the tangent points varies significantly - for the proposed constellation between $30 \mathrm{~km}$ in case of perpendicular orbits, up to $400 \mathrm{~km}$ in case of aligned orbits.

\section{Processing Strategy for Cross-Link Occultations}

The frequency residuals, as expected from cross-link occultations between main orbiter and the four cubesats in formation, allow for computation of the atmospheric state variables (pressure and temperature) along the path of signal propagation. However, the processing is not straight forward, but requires ancillary information (e.g., satellite ephemerides) and is based on assumptions concerning signal propagation and the atmospheric strata.

\subsection{Conventional Retrieval Method}

Ignoring diffraction effects and assuming spherical atmospheric symmetry, the frequency residuals may be processed using the Abel transform to obtain vertical profiles of index of refraction (Fjeldbo et al., 1971; Hinson et al., 1999; Withers et al., 2014), which can be further converted into temperature and pressure. Applied to cross-link occultations, this method will lead to vertical profiles of pressure and temperature with a resolution of about $500 \mathrm{~m}$ (limited by the Fresnel-scale) and thus, will provide a better insight into the vertical atmospheric structure during all local times scanned by the four cubesats in formation.

Several studies exists about possible extensions of the conventional retrieval method, for example, to further increase the vertical resolution by scalar diffraction theory (Karayel \& Hinson, 1997), However, the horizontal resolution remains a limiting factor. Due to the low density of the Martian atmosphere, solar heating and infrared cooling causes large temperature variations of more than $100 \mathrm{~K}$, which, in consequence, cannot be resolved by conventional retrieval methods, see Figure 5. Thus, in the following an alternative processing strategy for RO measurements is introduced.

\subsection{Tomographic Processing}

In order to overcome the limitations of the Abel transform - especially the symmetry assumption - and to further increase the horizontal resolution, we propose a new processing strategy which is based on tomographic principles. As shown in the following, tomographic principles are well suited for the processing of "clustered" RO measurements, as expected from dense cubesat formations. According to Iyer and Hirahara (1993), the general principle of tomography is described as follows:

$$
f_{s}=\int_{S} g(s) \cdot d s
$$



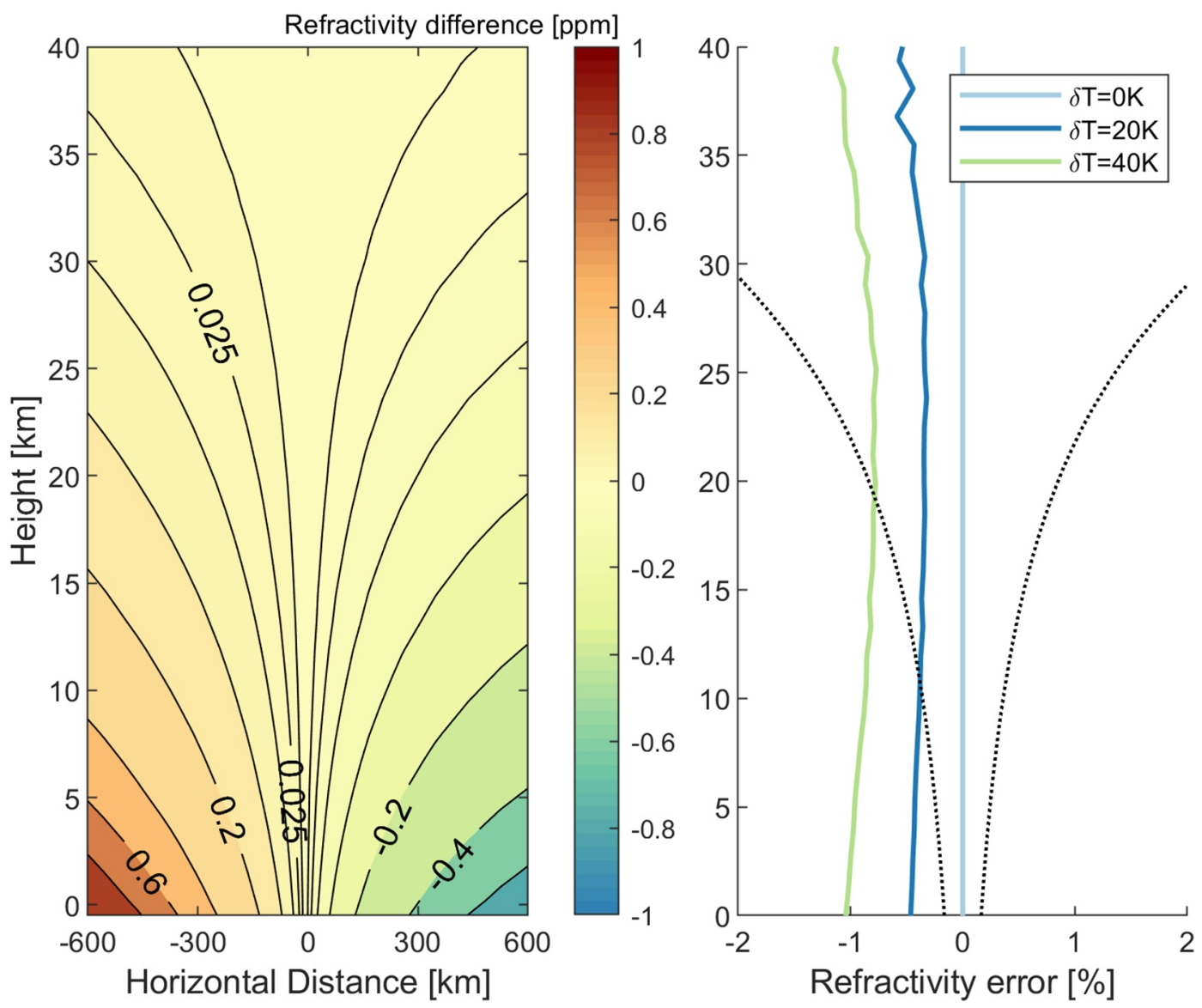

Figure 5. Left: Impact of a horizontal temperature change of $40 \mathrm{~K}$ on the refractivity structure in the lower $40 \mathrm{~km}$ of the atmosphere. Right: Resulting error in refractivity caused by the assumption of spherical atmospheric symmetry in the radio occultation (RO) signal processing - exemplary for a temperature change of 0,20 , and $40 \mathrm{~K}$. The dashed lines represent the typical $1-\sigma$ measurement uncertainty for RO retrievals.

where $f_{s}$ is the projection function, $g(s)$ is the object property function and $d s$ is a small element of the ray path $S$ along which the integration takes place. For the processing of RO data, $g(s)$ is replaced by index of refraction $n$ and $f_{s}$ is the signal phase delay $(d L)$. If Doppler shift $(d f)$ is provided instead of phase delay, with sampling rate $d t$, it can be converted as follows:

$$
d L=\sum_{i} \Delta d L(i)
$$

with

$$
\Delta d L(i)=-\frac{d f(i)}{1+f_{t} / f_{r}} \cdot \frac{c}{f_{r}} \cdot d t .
$$

In Equation 4, the correction term $\left(f_{t} / f_{r}\right)$ has to be applied if the received frequency $\left(f_{r}\right)$ differs from the transmitted frequency $\left(f_{t}\right)$, for example, if the transmitted signal is multiplied with a certain ratio before re-transmission, which is beneficial in the two-way RO concept. In any case, the ionospheric effect on the Doppler shift has to be corrected beforehand using dual-frequency observations or ionospheric models like the one described by Pi et al. (2008). The resulting basic function of tomography reads:

$$
d L=\int_{S} n \cdot d s-\int_{S_{0}} d s
$$


where $S$ is the "true" signal path and $S_{0}$ is the theoretical straight line signal path in vacuum.

One difficulty in performing the first integral in Equation 5 is that the ray path is not a straight line but rather dependent on the object properties along the signal path. A change in $n$ leads to a change in $S$ and $f_{s}$. However, from Fermat's principle it can be assumed that first order changes of the ray path lead to second order changes in travel time, that is, for small perturbation of the path, the travel time is stationary. In fact, we make use of this principle for setting up the tomography approach. The resulting "non-linear" approach ignores the path-dependency in the inversion of $n$ along $d s$ but takes the signal bending into account by the definition of the ray paths. In consequence, the tomography solution is derived iteratively. After each processing step the ray paths are re-computed by solving the Eikonal equation using, for example, ray-tracing shooting techniques, see Moeller and Landskron (2019).

In order to find a numerical solution for Equation 5, the object of interest, for example, the neutral atmosphere is discretized in area elements (in two-dimensions) in which the index of refraction is assumed as constant. Consequently and by replacing the index of refraction with refractivity $N=(n-1) \times 10^{6}$, Equation 5 reads:

$$
d L=10^{-6} \sum_{k=1}^{m} N_{k} \cdot d_{k}
$$

where $N_{k}$ is the refractivity and $d_{k}$ is the ray length in area element $k$.

In case of overlapping signal paths (e.g., in case of aligned orbital planes), a linear equation system can be set up for the reconstruction of the refractivity along the signal paths. In matrix notation it reads:

$$
\mathbf{d L}=\mathbf{A} \cdot \mathbf{N}
$$

where $\mathbf{d L}$ is the observation vector, $\mathbf{N}$ is the vector of unknowns and $\mathbf{A}$ is a matrix which contains the spatial derivatives of the observations with respect to the unknowns, that is, the ray lengths $\left(d_{k}\right)$ in each area element. For determining the unknown vector $\mathbf{N}$, the inverse $\mathbf{A}^{\mathbf{- 1}}$ must to be formed. However, in most cases matrix $\mathbf{A}$ is not of full rank, thus regularization methods have to be applied to determine the pseudo inverse. Therefore, we make use of truncated singular value decomposition methods as described by Strang and Borre (1997) and Moeller (2017).

\section{Tomography Case Study}

For technique demonstration, a closed-loop simulation was carried out using planetWRF (Richardson et al., 2007) - a modified version of the Weather Research and Forecasting (WRF) model for planetary atmospheres - to simulate the atmospheric state along the RO signal paths of the proposed cubesat-orbiter constellation. The model data used are atmospheric pressure and temperature, provided on a global $5 \times 5$ grid for 40 vertical layers, with a 3 -h temporal resolution. In a first step, the signal paths through the atmosphere were reconstructed every $500 \mathrm{~ms}$ using ray-tracing shooting techniques (Moeller \& Landskron, 2019) with a step size of $1 \mathrm{~km}$. Since atmospheric density is exponentially decreasing with altitude, refractivity $N$ was computed for signals penetrating into the lower $50 \mathrm{~km}$ of the atmosphere only. The simulated refractivities along the RO signal paths were converted into phase delays using Equation 6 and the area covered by the observations was parameterized in area elements with a grid size of $60 \mathrm{~km}$ (horizontally) $\times 1 \mathrm{~km}$ (vertically). Figure 6 shows the observation geometry together with the ray paths through the simulated refractivity field. For the case study, only observations with an azimuth angle less than $25^{\circ}$ were simulated. For these observations tomographic principles are most beneficial - mainly due to overlapping signal paths (see Figure 3 right). The tomographic processing itself, that is, the estimation of refractivity fields from phase measurements, was carried out using a modified version of the ATom software package (Moeller, 2017). Table 2 summarizes the major settings.

For visualization and validation against WRF, the tomography derived refractivity fields were converted into atmospheric pressure and temperature, assuming hydrostatic equilibrium and an initial temperature of $220 \mathrm{~K}$ at $50 \mathrm{~km}$ altitude. Figure 7 shows the resulting temperature field and temperature differences 

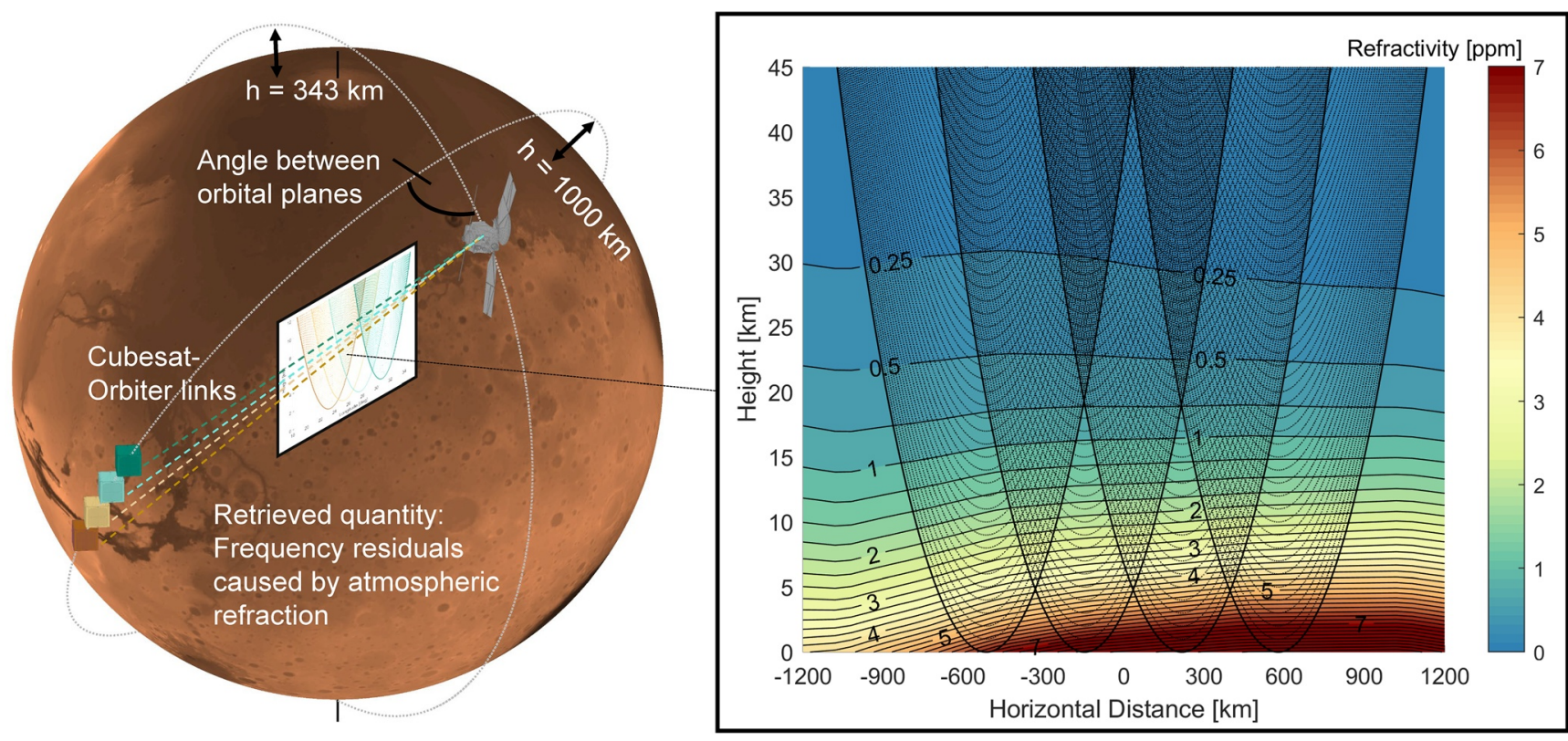

Figure 6. Left: Observation geometry for cross-link occultations. Right: The planetWRF (Richardson et al., 2007) derived refractivity field along the radio occultation signal paths.

for a case study in high northern latitudes $\left(40^{\circ}-80^{\circ} \mathrm{N}\right)$ in late autumn $\left(L_{s}=270^{\circ}\right)$. The WRF-based temperature field is characterized by strong vertical inversions and distinct negative horizontal temperature gradients in the direction of the North Pole. Figure 7 demonstrates that these temperature structures are very well resolvable by the proposed cubesat formation and processing of the RO signals using tomographic principles.

For the tomography derived temperature field, a Root Mean Square Error (RMSE) of $7.3 \mathrm{~K}$ and a bias of $1.4 \mathrm{~K}$ was obtained for the lowest $30 \mathrm{~km}$ of the atmosphere. Above, the RMSE increases - mainly due to temperature initialization problems. Overall, the best solution was obtained within the horizontal range $(-600 \mathrm{~km}, 600 \mathrm{~km})$ in which multiple observations overlap (see Figure 6 right) and therefore, help to further stabilize the tomography solution. The RMSE in this "core" domain of the tomography model is about $3.8 \mathrm{~K}$, and therefore, by a factor of 2-3 better than in the outer regions. In contrast, the solution based on Abel transform provides a slightly smaller bias of $0.5 \mathrm{~K}$, but with $11.3 \mathrm{~K}$ a $55 \%$ larger RMSE for the entire region, and with $10.9 \mathrm{~K}$ a $185 \%$ larger RMSE for the "core" domain of the tomography model.

Table 2

Tomography Settings Applied for the Reconstruction of Refractivity Fields From (Simulated) Cross-Link RO Observations

\begin{tabular}{lc}
\hline Parameter & Settings \\
\hline Case study domain & High northern latitudes $\left(40^{\circ}-80^{\circ} \mathrm{N}\right)$ \\
Case study period & Late autumn $\left(L_{s}=270^{\circ}\right)$ \\
Model resolution & $60 \mathrm{~km}$ (horizontally) $\times 1 \mathrm{~km}$ (vertically) \\
Tomography software & Modified version of ATom software package ${ }^{\mathrm{a}}$ \\
Initial field & Symmetric refractivity field obtained using Abel transform \\
Inversion method & Singular value decomposition (eigen $\left.v_{\text {min }}=0.01 \mathrm{~km}^{2}\right)$ \\
Estimation method & Iterative weighted least squares adjustment \\
Convergence criteria & RMS of weighted residuals \\
\hline${ }^{a}$ https://github.com/GregorMoeller/ATom &
\end{tabular}



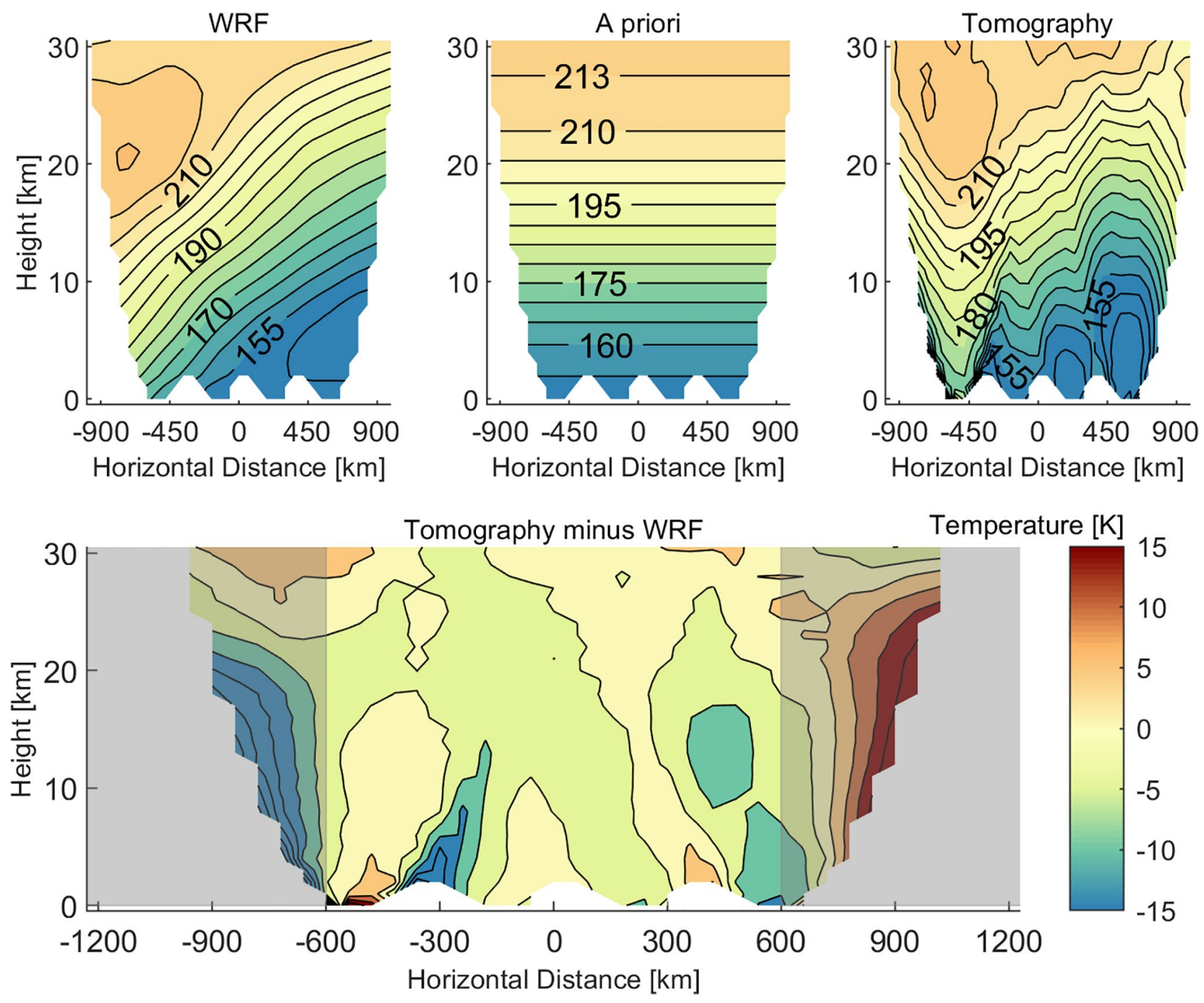

Figure 7. Top left: planetWRF temperature field for high northern latitudes in late autumn (reference). Top middle: Symmetric a priori field obtained using Abel transform. Top right: Estimated temperature field using tomographic principles. Bottom: Closed-loop validation (Tomography minus Weather Research and Forecasting) to assess the performance of the tomography approach under rather realistic atmospheric conditions.

\section{Conclusions and Outlook}

In this study, the basic aspects for the remote sensing of the Martian atmosphere using tomographic RO methods are addressed. Our motivation is to provide valuable measurements about the diurnal cycle and various meso- to small-scale processes in the lower Martian atmosphere. Both have been identified by the Integration Report from the 9th International Conference on Mars (Yingst et al., 2019) as the major gaps in the current Martian observing network. In contrast to existing cross-link occultation studies, the focus is on the sampling of specific regions in the lower Martian atmosphere with high spatial resolution - rather than a homogeneous distribution of RO events. Therefore, four cubesats in a "string-of-pearls" formation have been identified as minimum requirement. The challenge will be to maintain such kind of formation over a period of one Martian year at least - if possible without active propulsion systems. The details of orbit control, for example, using an intelligent attitude control system, have to be examined in further studies.

In a number of closed-loop validations, the expected observations but also possible processing strategies have been evaluated. Due to the unique observation geometry, a combined processing of the RO measurements using tomographic principles seems to be promising and allows to further increase the horizontal resolution 
of the reconstructed temperature and pressure fields. In addition, tomographic principles are not based on a spherical symmetry assumption and thus, allow for resolving distinct temperature gradients and inversion layers in the lower Martian atmosphere down to the surface - even during high opacity conditions, for example, during dust storms. In the conducted tomography case study, we have identified a core domain in which an accuracy in temperature of a few Kelvin is achieved. The extent of the domain but also the temperature accuracy might be further increased by additional cubesats added to the proposed constellation.

For realization of the occultation measurements, a dual one-way inter-satellite link between the cubesats and a main orbiter is suggested. The advantage of this concept is lower demands on the frequency stability but requires a dual-frequency radio on both sides, as well as active cubesats for signal tracking and data transfer. Further details about the satellite-to-satellite tracking system can be found in Kim and Tapley (2002). The necessary modifications for the proposed cubesat formation will be described in a subsequent report.

\section{Data Availability Statement}

The scripts and input data for orbit prediction as well as the data set for the tomography test case are available at https://doi.org/10.3929/ethz-b-000441288.

\section{References}

\section{Acknowledgments}

This work was performed at the Jet Propulsion Laboratory, California Institute of Technology, under a contract with National Aeronautics and Space Administration (NASA). Gregor Moeller's research study was supported by an appointment to the NASA Postdoctoral Program at NASA Jet Propulsion Laboratory, administered by Universities Space Research Association under contract with NASA. The authors gratefully acknowledge Michael A. Mischna (NASA, JPL) for providing the planetWRF data.
Ao, C. O., Edwards, C. D., Kahan, D. S., Pi, X., Asmar, S. W., \& Mannucci, A. J. (2015). A first demonstration of Mars crosslink occultation measurements. Radio Science, 50(10), 997-1007. https://doi.org/10.1002/2015RS005750

Asmar, S. W., Ao, C. O., Edwards, C. D., Kahan, D. S., Pi, X., Paik, M., \& Mannucci, A. J. (2016). Demonstration of Mars crosslink occultation measurements for future small spacecraft constellations. In 2016 IEEE Aerospace Conference. (pp. 1-6). https://doi.org/10.1109/ AERO.2016.7500729

Asmar, S. W., Lazio, J., Atkinson, D. H., Bell, D. J., Border, J. S., Grudinin, I. S., et al. (2019). Future of planetary atmospheric, surface, and interior science using radio and laser links. Radio Science, 54(4), 365-377. https://doi.org/10.1029/2018RS006663

Fjeldbo, G., Kliore, A. J., \& Eshleman, V. R. (1971). The neutral atmosphere of Venus as studied with the Mariner V radio occultation experiments. The Astronomical Journal, 76(2), 123. https://doi.org/10.1086/111096

Hinson, D. P., Simpson, R. A., Twicken, J. D., Tyler, G. L., \& Flasar, F. M. (1999). Initial results from radio occultation measurements with Mars Global Surveyor. Journal of Geophysical Research, 104(E11), 26997-27012. https://doi.org/10.1029/1999JE001069

Iyer, H., \& Hirahara, K. (1993). Seismic tomography: Theory and practice. Springer Netherlands.

Joffre, E., Derz, U., Perkinson, M.-C., Huesing, J., Beyer, F., \& Sanchez Perez, J.-M. (2018). Mars Sample Return: Mission analysis for an ESA Earth Return Orbiter. In 7th International Conference on Astrodynamics Tools and Techniques (ICATT). (pp. 1-15).

Karayel, E. T., \& Hinson, D. P. (1997). Sub-fresnel-scale vertical resolution in atmospheric profiles from radio occultation. Radio Science, 32(2), 411-423. https://doi.org/10.1029/96RS03212

Kim, J., \& Tapley, B. D. (2002). Error analysis of a low-low satellite-to-satellite tracking mission. Journal of Guidance, Control, and Dynamics, 25(6), 1100-1106. https://doi.org/10.2514/2.4989

Kliore, A., Cain, D. L., Levy, G. S., Eshleman, V. R., Fjeldbo, G., \& Drake, F. D. (1965). Occultation experiment: Results of the first direct measurement of Mars's atmosphere and ionosphere. Science, 149(3689), 1243-1248. https://doi.org/10.1126/science.149.3689.1243

Mannucci, A. J., Ao, C. O., Asmar, S., Edwards, C. D., Kahan, D. S., Paik, M., \& Williamson, W. (2015). Crosslink radio occultation for the remote sensing of planetary atmospheres. AGU Fall Meeting.

Moeller, G. (2017). Reconstruction of 3D wet refractivity fields in the lower atmosphere along bended GNSS signal paths (PhD thesis). TU Wien, Department of Geodesy and Geoinformation.

Moeller, G., \& Landskron, D. (2019). Atmospheric bending effects in GNSS tomography. Atmospheric Measurement Techniques, 12(1), 23-34. https://doi.org/10.5194/amt-12-23-2019

Pi, X., Edwards, C., Hajj, G., Ao, O., Romans, L., Callas, J., \& Kahan, D. (2008). A Chapman-layers ionospheric model for Mars. NASA STI/ Recon Technical Report N.

Richardson, M., Toigo, A., \& Newman, C. (2007). PlanetWRF: A general purpose, local to global numerical model for planetary atmospheric and climate dynamics. Journal of Geophysical Research, 112. https://doi.org/10.1029/2006JE002825

Strang, G., \& Borre, K. (1997). Linear algebra, geodesy, and GPS. Wellesley-Cambridge Press.

Tan, Z., Bainum, P. M., \& Strong, A. (2002). The implementation of maintaining constant distance between satellites in coplanar elliptic orbits. Journal of the Astronautical Sciences, 50, 53-69. https://doi.org/10.1007/bf03546330

Tellmann, S., Pätzold, M., Häusler, B., Bird, M. K., Hinson, D. P., Andert, T. P., \& Asmar, S. W. (2019). Crosslink occultations for probing the planetary atmosphere and ionosphere of Mars. (p. 2089). EPSC-DPS Joint Meeting.

Turk, F. J., Padulles, R., Ao, C. O., de la Torre Juarez, M., Wang, K.-N., Franklin, G. W., et al. (2019). Benefits of a closely-spaced satellite constellation of atmospheric polarimetric radio occultation measurements. Remote Sensing, 11(20), 2399. https://doi.org/10.3390/ rs11202399

Withers, P., Felici, M., Mendillo, M., Moore, L., Narvaez, C., Vogt, M. F., et al. (2020). The MAVEN Radio Occultation Science Experiment (ROSE). Space Science Reviews, 61(216), 49. https://doi.org/10.1007/s11214-020-00687-6

Withers, P., Moore, L., Cahoy, K., \& Beerer, I. (2014). How to process radio occultation data: 1. From time series of frequency residuals to vertical profiles of atmospheric and ionospheric properties. Planetary and Space Science, 101, 77-88. https://doi.org/10.1016/j.pss. 2014.06.011

Yingst, R. A., Forget, F., Calvin, W., Des Marais, D., \& Niles, P. (2019). Integration Reports from the Ninth International Conference on Mars. (Technical Report). 